\title{
A Comparison of Trace-Sampling Techniques for Multi-Megabyte Caches
}

\author{
R. E. Kessler, Mark D. Hill, and David A. Wood
}

\begin{abstract}
This paper compares the trace-sampling techniques of set sampling and time sampling. Using the multi-billionreference traces of Borg et at., we apply both techniques to multi-megabyte caches, where sampling is most valuable. We evaluate whether either technique meets a $10 \%$ sampling goal: a method meets this goal if, at least $90 \%$ of the time, it estimates the trace's true misses per instruction with $\leq 10 \%$ relative error using $\leq 10 \%$ of the trace. Results for these traces and caches show that set sampling meets the $10 \%$ sampling goal, while time sampling does not. We also find that cold-start bias in time samples is most effectively reduced by the technique of Wood et al. Nevertheless, overcoming cold-start bias requires tens of millions of consecutive references.
\end{abstract}

Index Terms-Cache memory, cache performance, cold start, computer architecture, memory systems, performance evaluation, sampling techniques, trace-driven simulation.

\section{INTRODUCTION}

C OMPUTER designers commonly use trace-driven simulation to evaluate alternative CPU caches [21]. But as cache sizes reach one megabyte and more, traditional tracedriven simulation requires very long traces (e.g., billions of references) to determine steady-state performance [4], [22]. But long traces are expensive to obtain, store, and use.

We can avoid simulating long traces by using tracesampling techniques. Let the cache performance of a small portion of the trace be an observation and a collection of observations be a sample. Sampling theory tells how to predict cache performance of the full trace, given a sample of unbiased observations [5], [14]. With additional assumptions, we can also estimate how far the true value is likely to be from the estimate.

Two important trace-sampling techniques are set sampling [7], [18] and time sampling [12], [13]. An observation in set sampling is the cache performance for the references to a single set (depicted as a horizontal slice in Fig. 1), while an observation in time sampling is the cache performance of the references in a single time-contiguous trace interval (a

Manuscript received September 31, 1991; revised June 1993. R. E. Kessler was supported in part by a summer internship at Digital Equipmen Corporation and graduate fellowships from the National Science Foundation and the University of Wisconsin Alumni Research Foundation. M. D. Hill was supported in part by the National Science Foundation (MIPS-8957278 and CCR-8902536), A.T.\& T. Bell Laboratories, Cray Research Foundation and Digital Equipment Corporation. D. A. Wood was supported in part by the National Science Foundation (CCR-9157366) and the University of Wisconsin Graduate School.

The authors are with the University of Wisconsin, Computer Sciences Department, Madison, WI 53706 USA; e-mail: \{kessler, markhill, david\} @cs.wisc.edu.

IEEE Log Number 9401099.

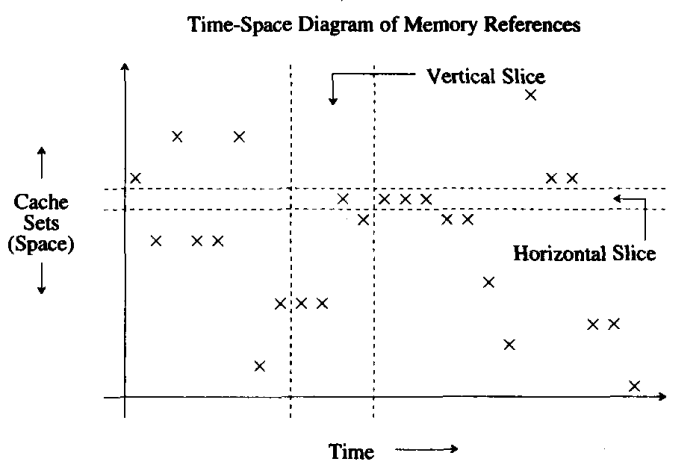

Fig. 1. Sampling as vertical and horizontal time-space slices. This figure shows a time-space diagram of a simulation with a very short trace.The time (position within the trace) and cache set of each reference is marked with an $x$. An observation in set sampling is the cache performance of one set. References that determine a single set's performance appear inan horizontal slice of this figure.An observation in time sampling is the cache performance of an intervalof consecutive references. These references appear in a vertical slice of this figure.

vertical slice in Fig. 1). Laha et al. [12] and Wood et al. [25] referred to an observation of references in a time-contiguous interval as a "sample." We use sample to refer to a collection of observations to be consistent with statistics terminology [14].

This study is the first to compare set sampling and time sampling. We use eight multi-billion-reference traces of large workloads that include multiprogramming but not operating system references [4], and concentrate on multi-megabyte caches, where sampling is most needed. For each trace and cache, we examine how well set and time samples from a trace predict the misses per instruction (MPI) of the entire trace. We say a sampling method is effective if it meets the $10 \%$ sampling goal: a method meets this goal if, at least $90 \%$ of the time, it estimates the trace's true misses per instruction with $\leq 10 \%$ relative error using $\leq 10 \%$ of the trace.

It is critical that readers note that the $10 \%$ sampling goal evaluates using samples from a trace to estimate that trace's MPI. We do not formally address how our traces relate to the population of all traces, because we know of no research that quantitatively characterizes that population. Like most tracedriven studies, we describe our traces and leave it to the reader to decide whether they are representative of a larger population. Section II-D discusses the reasoning behind the $10 \%$ sampling goal in more detail.

With our traces and caches, we find several results pertaining to set sampling (Section III). First, how we compute MPI is important. We find that it is much less accurate to 
normalize misses by the instruction fetches to the sampled sets than by the fraction of sampled sets times all instruction fetches. Second, instead of selecting the sets in a sample at random, selecting sets that share several index bit values reduces simulation time, facilitates the simulation of cache hierarchies, and still accurately predicts the trace's MPI. Third, and most important, set sampling is effective. For our traces and caches, it typically meets the $10 \%$ sampling goal.

For time-sampling (Section IV), we first compare techniques for overcoming cold-start bias [6], i.e., determining the MPI for a particular trace interval without knowing the initial cache state. We consider leaving the cold-start bias unchanged, recording metrics only during the second half of each interval, recording metrics only for initialized sets [12], [22], stitching intervals together [1], and Wood et al.'s model for predicting the initialization reference miss ratio [25]. For our traces and caches, we obtain two results. First, on average, the technique of Wood et al. minimizes the cold-start bias better than the other techniques. Second, for the multi-megabyte caches we studied, interval lengths of tens of millions of instructions and larger are needed to reduce the effects of cold-start.

Then using Wood et al.'s technique to mitigate cold-start bias, we find that time sampling fails to meet the $10 \%$ sampling goal for our traces and caches, because: 1) many intervals are needed to capture workload variation, and 2) long intervals are necessary to overcome cold-start bias. As a result, for these traces and caches, set sampling is more effective than time sampling for estimating MPI. Set sampling is not appropriate, however, for caches with time-dependent behavior (e.g., prefetching) or interactions between sets (e.g., a single write buffer).

We do not consider other (non-sampling) techniques that reduce trace data storage, such as, Mache [19], stack deletion and snapshot method [20], trace (tape) stripping [18], [23], or exploiting spatial locality [2]. These techniques can be used in addition to the sampling considered in this study. We also do not consider Przybylski's prefix technique [16], which prepends all previously-referenced unique addresses to each time-observation. This method seems unattractive for multimegabyte caches where each time-observation requires its own prefix and each prefix must be very large for programs that can exercise multi-megabyte caches.

\section{Methodology}

This section describes the traces, caches, and performance metric we use in later sections.

\section{A. The Traces}

The traces used in the study were collected at DEC Western Research Laboratory (WRL) [3], [4] on a DEC WRL Titan [15], a load/store ("RISC") architecture. Each trace consists of the execution of three to six billion instructions of large workloads, including multiprogramming but not operating system references. The traces of the multiprogrammed workloads represent the actual execution interleaving of the processes on the traced system. The traces reference from eight to over one hundred megabytes of unique memory locations. These traces are sufficiently long to overcome the cold-start
TABLE I A Description of THE STUdifo WorkLoAdS

\begin{tabular}{|c|c|}
\hline Workload & Description \\
\hline Mult I & 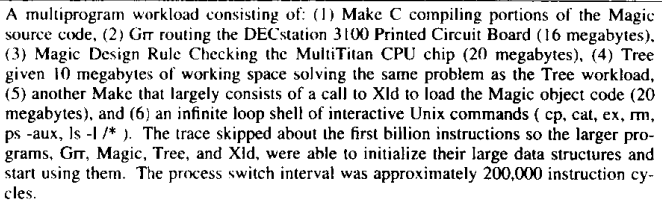 \\
\hline Mult1.2 & $\begin{array}{l}\text { The same workload as Mult l except the process switch interval is approximately } 400,000 \\
\text { hasic instruction cycles. }\end{array}$ \\
\hline Muth2 & $\begin{array}{l}\text { The MultI workbad excluding the XId (Make) run (5) and the Tree program (4). Mult2 } \\
\text { has a lower degree of multiprogramming and is smaller than Mult1. }\end{array}$ \\
\hline Mulli2.2 & $\begin{array}{l}\text { The same workload as Mult } 2 \text { except the process switch interval is approximately } 400,000 \\
\text { basic instruction cycles. }\end{array}$ \\
\hline$T v$ & $\begin{array}{l}\text { A uniprogram workload of TV analyzing the timing of the DEC WRL MultiTitan CPU } \\
\text { chip. Tv required } 12.5 \text { billion instructions to complete the timing analysis. About the } \\
\text { first } 10 \text { billion instruction build a very large linked data structure. The final } 2-3 \text { billion } \\
\text { instructions traverse the sitructure. The end of the execution of Tv was cappured on tape. }\end{array}$ \\
\hline Sor & $\begin{array}{l}\text { A uniprogram workload of the Sor program doing matrix manipulations on a } 800,000 \text { by } \\
200,000) \text { sparse matrix with approximately } 4 \text { million }(0.0025 \%) \text { of the matrix entries be- } \\
\text { ing non-zero. About the first billion instructions create the large matrices. The rest of } \\
\text { the program is the matrix operations. The trace captures a portion of the matrix opera- } \\
\text { tions, excluding initialization. }\end{array}$ \\
\hline Tree & $\begin{array}{l}\text { A uniprogram workload consisting of the Tree program. Tree has two major phases that } \\
\text { were traced. About the first half of the instructions build a large tree structure that } \\
\text { represents a Unix-like hierarchical directory structure. The rest of the instructions search } \\
\text { this tree to find the largest member. }\end{array}$ \\
\hline Lin & $\begin{array}{l}\text { A uniprogram workload of Linear analyzing the power supply of a register file. Normal- } \\
\text { ly, the program tries to minimize the amount of work it must do by combining circuit } \\
\text { structures. The trace was collected hy disabling some of these combining operations to } \\
\text { produce a bigger problem, possibly reflecting the larger prohlerms of the future. }\end{array}$ \\
\hline
\end{tabular}

This table consists of a description of the user-only (no kernel references) workloads used in this study. Four workloads are uniprogrammed and two are multiprogrammed workloads. The uniprogrammed workloads consist of the largest programs. Several smaller programs were grouped with some standard Unix programs to produce the multiprogrammed workloads.

intervals of even the large caches considered in this study. We chose programs with large memory requirements because of the likelihood that large application sizes will become more common as main memories of hundreds of megabytes become available.

Table I describes traces in detail. The Mult 2 trace includes a series of compiles, a printed circuit board router, a VLSI design rule checker, and a series of simple programs commonly found on UNIX ${ }^{\mathrm{TM}}$ systems, all executing in parallel (about 40 megabytes active at any time) with an average of 134000 instructions executed between each process switch. The Mult2.2 trace is the Mult2 workload with a switch interval of 214000 instructions. The Mult1 trace includes the processes in the Mult 2 trace plus an execution of the system loader (the last phase of compilation) and a Scheme (Lisp variant) program ( 75 megabytes active) and has a switch interval of 138000 instructions. The Mult1.2 trace is the Mult l workload with a switch interval of 195000 instructions. The Tv trace is of a VLSI timing verifier (96 megabytes). Sor is a uniprocessor successive-over-relaxation algorithm that uses large, sparse matrices (62 megabytes). Tree is a Scheme program that searches a large tree data structure $(64$ megabytes). Lin is a power supply analyzer that uses sparse matrices (57 megabytes).

\section{B. Cache Assumptions}

This study focuses on multi-megabyte unified (both instructions and data cached together) caches. Earlier work has shown

\footnotetext{
${ }^{\text {TM }}$ Trademark AT\&T Bell I.aboratories.
} 
that both techniques are effective for smaller caches [12], [18]. We vary the size and set-associativity of these caches over a range of sizes from 1-megabyte to 16-megabytes and associativities from direct-mapped to four-way. The caches do no prefetching, use write-back and write-allocate policies, and have 128-byte blocks. The caches use virtual-indexing (i.e., select the set of a reference using the reference's virtual address) with PID-hashing, an approximation to real-indexing. PID-hashing means that we exclusive-or the upper eight index bits from the virtual address with the process identifier (PID) of the currently executing process. We also examined several real-indexed caches and found that they produced results similar to those in this paper, which is not surprising since real-indexed cache performance is often close to virtualindexed cache performance. The non-direct-mapped caches use a random replacement policy, which was easier to handle in the our simulation environment than is least-recently-used replacement.

Since multi-megabyte caches are likely to be used in a cache hierarchy, we simulate them as alternative secondary caches placed behind a fixed primary cache. The primary caches are split (separate) instruction and data caches that are 32-kilobytes each, direct-mapped, 32-byte blocks, do no prefetching, use virtual indexing, and write-back and writeallocate policies. We do not evaluate primary cache tradeoffs in this study since secondary cache performance is unaffected by the primary caches when their sizes differ by at least a factor of eight [17].

\section{The Performance Metric: Misses Per Instruction}

We measure cache performance with misses per instruction (MPI) rather than miss ratio. For comparing the performance of alternative unified secondary caches, MPI is equivalent to Przybylski's global miss ratio [17]. Specifically, a cache's MPI is equal to its global miss ratio times the average number of processor references (instruction fetches and data references) per instruction.

\section{The $10 \%$ Sampling Goal}

Given a particular trace and cache, let MPI $_{\text {true }}$ be MPI obtained by simulating the complete trace with an initially empty cache (the true MPI of the complete trace). We say a sampling method is effective (for that trace and cache) if it meets the following goal:

Definition 1) 10\% Sampling Goal: A method meets the $10 \%$ sampling goal if, at least $90 \%$ of the time, it estimates the trace's true misses per instruction with $\leq 10 \%$ relative error using $\leq 10 \%$ of the trace.

The 10\% sampling goal evaluates using samples from a trace to estimate MPI $_{\text {true }}$ (that trace's MPI). As discussed in the introduction, we do not formally address how our traces relate to the population of all traces, because we know of no research that quantitatively characterizes that population. For this reason, readers must choose between accepting our results (by assuming our traces are representative of their workload) and re-applying our techniques to their traces. We share this failure of generalizing to the population of all traces with all trace-driven cache studies we are aware of.

We chose $\leq 10 \%$ of the references in a trace and $\leq 10 \%$ relative error using our experience with cache design and evaluation. We expect cache designers would not confront the intellectual complexity of sampling for less than a factor of ten reduction in trace size. We also expect many cache designers would consider negligible a $10 \%$ relative error in estimating a trace's MPI, since MPI variations between traces often exceed factors of ten. Nevertheless, other cache designers may wish to choose stricter or looser criteria and re-apply the techniques described in this paper.

\section{Set SAmPLing}

We first examine set sampling, where an observation is the MPI of a single set and a sample is a collection of singleset observations. Section III-A discusses how to compute a set sample's MPI and why it should not contain random sets, while Section III-B examines how well set sampling predicts $\mathrm{MPI}_{\text {true }}$, the MPI of a full trace.

\section{A. Constructing Set Samples}

Calculating the MPI of a Sample: In this section, we find that how we compute MPI is important; specifically, it is much less accurate to normalize misses by the instruction fetches to the sampled sets than by the fraction of sampled sets times all instruction fetches. Consider a cache with $s$ sets. For each set $i$, let miss ${ }_{i}$ and instrn ${ }_{i}$ be the number of the misses and instruction fetches to set $i$. Let $\mathbf{S}$ be a sample containing all references to $n$ sets.

We consider two ways to calculate the MPI of sample $\mathbf{S}$. $\widehat{\mathrm{MPI}}_{S}$, which both require two counters to process a trace. Both use one counter to accumulate the number of the misses to the sets in the sample. At the end of the trace, this counter equals $\sum_{i \in \mathbf{S}}$ miss $_{i}$.

The sampled-instructions method uses the second counter to accumulate the instruction fetches to the sets in the sample $\left(\sum_{i \in \mathbf{S}}\right.$ instrn $\left._{i}\right)$ and computes $\widehat{\mathrm{MPI}}_{S}$ with:

$$
\widehat{\mathrm{MPI}}_{S}=\frac{\sum_{i \in \mathrm{S}} \text { miss }_{i}}{\sum_{i \in \mathrm{S}} \text { instrn }_{i}} .
$$

The fraction-instructions method uses the second counter to to accumulate the instruction fetches to all sets in the cache $\left(\sum_{i=1}^{s} \operatorname{instrn}_{i}\right)$ and computes $\widehat{\mathrm{MPI}}_{S}$ with:

$$
\widehat{\mathrm{MPI}}_{S}=\frac{\sum_{i \in \mathbf{S}} \operatorname{miss}_{i}}{\frac{n}{s} \sum_{i=1}^{s} \text { instrn }_{i}} .
$$

An alternative view of the effort required for these two methods is to consider the information that must be saved from the full trace if cache simulation is not done when gathering the trace. Both methods require that all references to the sampled sets be saved. The fraction-instructions method also requires a count of the number of instruction fetches in the full trace. Since most trace-gathering tools accommodate adding a counter, we consider the difficulty of obtaining data for the two methods comparable. 
TABLE II

COEFFICIENT OF VARIATION OF MPI COMPUTATIONS

\begin{tabular}{|c|c|c|c|}
\hline Trace & $M P I_{\text {true }} \times 1060$ & $\begin{array}{c}\text { Coefficient of } \\
\text { fraction-instructions }\end{array}$ & $\begin{array}{l}\text { riation (percent) } \\
\text { sampled-instructions }\end{array}$ \\
\hline Mult 1 & 0.70 & $2.3 \%$ & $35.2 \%$ \\
\hline Multi.2 & 0.69 & $1.9 \%$ & $28.9 \%$ \\
\hline Mult2 & 0.61 & $1.9 \%$ & $24.2 \%$ \\
\hline Mult2.2 & 0.59 & $1.3 \%$ & $24.3 \%$ \\
\hline $\mathrm{TV}$ & 1.88 & $0.6 \%$ & $139.0 \%$ \\
\hline Sor & 7.54 & $0.3 \%$ & $\infty$ \\
\hline Tree & 0.59 & $6.8 \%$ & $191.9 \%$ \\
\hline Lin & 0.09 & $7.6 \%$ & $\infty$ \\
\hline
\end{tabular}

This table illustrates the accuracy of computing the full trace MPI (column (wo) for several traces with the fraction-instructions and sampled-instructions methods. The accuracy is evaluated with the coefficient of variation (Equation (1) for the MPI estimates from a 4-megabyte direct-mapped secondary cache with 16 set samples of $1 / 16$ the full trace each. The set samples are constructed with the constant bits method described in the next section. Results show that the fraction-instructions method is far superior to the sampled-instructions method

In addition, statistics for the fraction-instructions method are simpler than for sampled-instructions method. Since the fraction-instructions method normalizes the number of misses by a constant (for a given sample size $n$ and number of sets $s$ ), its MPI estimates can be handled as simple random variables. MPI estimates for the sampled-instructions method, on the other hand, should be modeled as the ratio of two random variables.

We empirically compare the two methods by computing their coefficients of variation across all set samples $S(j)$ obtained with the constant-bits method, a systematic sampling method described in Section III-A-2:

$$
\mathrm{CV}=\frac{\sqrt{\frac{1}{k} \sum_{j=1}^{k}\left(\widehat{\mathrm{MPI}}_{S(j)}-\mathrm{MPI}_{\mathrm{true}}\right)^{2}}}{\mathrm{MPI}_{\text {true }}},
$$

where $k=\frac{s}{n}$ is the number of samples [5, p. 208]. CV is the true coefficient of variation, because we compare the MPI's of all set samples from the finite population with the trace's true MPI. We do not compare the methods with expected error, $\frac{1}{k} \sum_{j=1}^{k}\left(\widehat{\mathrm{MPI}}_{S(j)}-\mathrm{MPI}_{\text {true }}\right)$, because the expected error of all set samples from the finite population is always zero.

Experimental results, illustrated in Table H, show that the fraction-instructions method performs much better, never having a coefficient of variation more than one-tenth the sampled-instructions method. The difference is infinite for the Sor and Lin traces because loops confine many instruction fetches to a few sets.

We also investigated normalizing miss ${ }_{i}$ with total references per set and data references per set [10]. These methods perform similarly to the sampled-instructions method and not as well as the fraction-instructions method. We did not consider calculating $\widehat{\mathrm{MPI}}_{S}$ with $\frac{1}{n} \sum_{i \in \mathrm{S}} \frac{\text { miss }_{i}}{\text { instrn }_{i}}$, because Puzak [18] showed estimating miss ratio with the arithmetic mean of the per-set miss ratios is inferior to dividing the misses to sampled sets by the references to sampled sets (the miss-ratio equivalent of the sampled-instructions method). For a sample containing all sets, Puzak's work also implies $\frac{1}{s} \sum_{i=1}^{s} \frac{\text { miss }_{i}}{\text { instrn }_{2}} \neq \mathrm{MPI}_{\text {true }}$

Since the fraction-instructions method performed better than the other methods examined, we will use it for the obtaining the remaining set sampling results.

The Constant-Bits Method: We now examine two methods for selecting sets to form a sample. We show why systematic

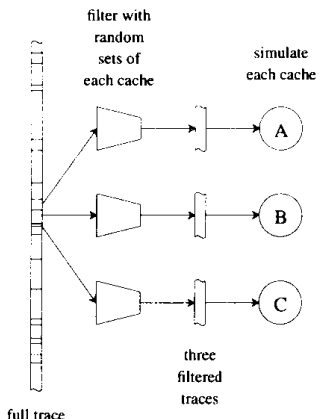

(a)

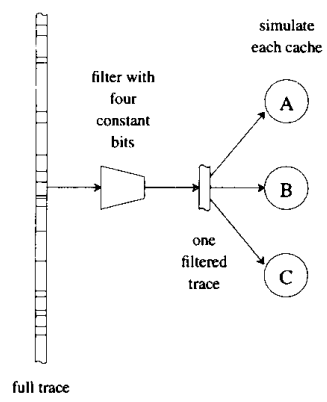

(b)
Fig. 2. Two methods for selecting the sets in a sample. This figure illustrates selecting sets for samples of three alternative caches (A, B, and C) using (a) random sets and (b) constant bits. When sets are selected at random, each simulation must begin by filtering the full trace. With constant-bits, on the other hand, a filtered trace can drive the simulation of any cache whose index bits contain the constant bits.

samples constructed via constant-bits offer advantages over random samples.

Assume that we want to evaluate three caches with samples that contain about $1 / 16$ th the references in a full trace. Let the caches choose a reference's set with bit selection (i.e., the index bits are the least-significant address bits above the block offset) and have the following parameters:

Cache A: 32-kilobyte direct-mapped cache with 32-byte blocks (therefore its index bits are bits 14-5, assuming references are byte addresses with bit 0 being least-significant); Cache B: 1-megabyte two-way set-associative cache with 128-byte blocks (index bits 18-7); and

Cache C: 16-megabyte direct-mapped cache with 128-byte blocks (index bits 23-7).

One method for selecting the sets in a sample is to choose them at random [18]. To evaluate cache $A$ with references to random sets, we randomly select 64 of its 1024 sets (1/16th), filter the full trace to extract references to those sets, and then simulate cache $A$. For cache $B$, we select 128 of its 2048 sets, filter and simulate. Similarly for cache $C$, we use 8192 of its 131072 sets. As illustrated in Fig. 2(a), selecting sets at random requires that each simulation begin by extracting references from the full trace. Furthermore, since primary and secondary caches usually have different sets, it is not clear how to simulate a hierarchy of cache when sets are selected at random.

A second method, which we call constant-bits, selects references rather than sets $[22$, p. 59]. The constant-bits method forms a filtered trace that includes all references that have the same value in some address bits. This filtered trace can then be used to simulate any cache whose index bits include the constant bits $^{2}[10]$. For example, if we filter a trace by retaining all references that have the binary value

2This description assumes bit selection, i.e., the set-indexing bits come directly from the address of the memory access [21]. The scenario is more complicated with other than simple bit-selection cache indexing. In particular. since we use PID-hashing in this study, we ensured that the hashed index bits did not overlap with the constant bits. Note that though we use virtualindexing, one can apply the constant-bits technique to real-indexed caches. and to hierarchical configurations with both real and virtual indexed caches if the constant bits are below the page boundary. 


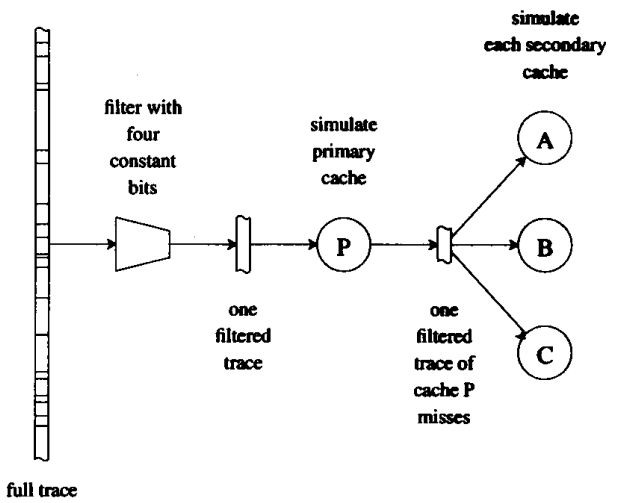

Fig. 3. Using constant-bits samples with a hierarchy. This figure illustrates how to use constant-bits samples to simulate a primary cache $(\mathrm{P})$ and three alternative secondary caches $(A, B$ and $C$ ).

0000 (or one of the other 15 values) in address bits $11-8$, then we can then use the filtered trace to select 1/16-th of the sets in any cache whose block size is 256 bytes or less and whose size divided by associativity exceeds 2 kilobytes. These caches include caches $A, B$, and $C$, the primary caches used in this study (32-byte blocks, 32 kilobytes, direct-mapped) and all secondary caches (128-byte blocks, 1-16 megabytes, 1-4-way set-associative) considered in this paper.

Constant-bits samples have two advantages over random samples. First, as illustrated in Fig. 2(b), using constantbits samples reduces simulation time by allowing a filtered trace to drive the simulations of more than one alternative cache. Second, constant-bits samples make it straightforward to simulate hierarchies of caches (when all caches index with the constant bits). As illustrated in Fig. 3, we may simulate the primary cache once and then use a trace of its misses to simulate alternative secondary caches.

One complexity of using constant-bits samples is that they are not random samples, since sets are selected systematically via certain bit patterns. Intuitively, constant-bits samples may work better than random samples if spreading the sampled sets throughout the cache captures more workload variation than selecting random sets. Constant-bits samples could perform worse than random samples, however, for workloads that use their address space systematically (e.g., frequent accesses to a large, fixed stride vector).

Cochran [5, ch. 8] develops a theory of systematic samples, which we review in Appendix A. Since the sample mean for both random and systematic samples are unbiased estimates of the population mean, systematic sampling yields more accurate estimates of the population mean if and only if the variance of the systematic sample mean is less than the variance of a random sample mean.

We examine this empirically in Table III. For each trace with a 4-megabyte, direct-mapped cache, Table III displays the variance of the random sample mean divided by the variance of the systematic sample mean obtained with the constant bits method. Values greater than one indicate that systematic samples are more accurate than random samples; we see that systematic samples are more accurate or comparable to random samples in all cases.
TABLE III

Random SAMPLE Variance over SYSTEMatic SAMPLE Variance

\begin{tabular}{|c|c|c|c|}
\hline Trace & $M P I_{\text {true }} \times 1000$ & \multicolumn{2}{|c|}{$\begin{array}{l}\text { Fraction of Sets in Sample } \\
1 / 16\end{array}$} \\
\hline Mult1 & 0.70 & 1.78 & 1.71 \\
\hline Mult1.2 & 0.69 & 1.39 & 1.27 \\
\hline Mult2 & 0.61 & 1.02 & 1.80 \\
\hline Mult2.2 & 0.59 & 1.85 & 1.96 \\
\hline Tv & 1.88 & 1.94 & 0.74 \\
\hline Sor & 7.54 & 26.05 & 19.45 \\
\hline Tree & 0.59 & 0.94 & 0.99 \\
\hline Lin & 0.09 & 1.26 & 1.35 \\
\hline
\end{tabular}

For each trace with a 4-megabyte, direct-mapped cache, this table shows the variance of the sample mean from a random sample (Equation (Al) in Appendix A) divided by the variance of the sample mean from a systematic sample mean (Equation (A2)). Systematic samples for $1 / 16$ (1/64) contain all references to sets with a fixed value in bits 11-8 (12-7). Values greater than one indicate that systematic samples more accurately predict $\mathbf{M P I}_{\mathbf{l} \text {.rue }}$ than do random samples.

Since the constant-bits method is easier to use than random samples and provides similar or better precision, we use the constant-bits method to construct set samples throughout the rest of this paper.

\section{B. What Fraction of the Full Trace is Needed?}

This section examines how well set samples estimate the MPI of a full trace. For reasons discussed above, we construct samples with the constant-bits method and calculate MPI estimate for a sample with the fraction-instructions method. We first look at the accuracy of set sampling when MPI true $_{\text {is }}$ known; then we show how to construct confidence intervals for MPI true when it is not known.

Table IV quantifies the error between set samples and MPI $_{\text {true }}$ for several traces, direct-mapped cache sizes, and sample sizes. We measure errors with coefficient of variation calculated using (1). Table $X$ in Appendix $C$ gives the corresponding results for two-way set-associative caches.

The key result is that, for this data and for four-way setassociative caches not shown here, set sampling generally meets the $10 \%$ sampling goal. Consider the columns labeled "1/16" in Tables IV and X, which correspond to samples using $1 / 16$ th of the sets and therefore will contain less than $10 \%$ of the trace on average. Only Lin and Tree with 4-megabyte direct-mapped caches, marked with daggers, fail to have at least $90 \%$ of the constant-bits samples with relative errors of less than or equal to $\pm 10 \%$. (And they both have only 2 of 16 samples with more than $\pm 10 \%$ relative error.) The data also show the set sampling performs well even if $1 / 64$ th of the sets are sampled.

We also observe that increasing associativity from directmapped to two-way reduces corresponding coefficients of variation by more than $50 \%$. We conjecture that set sampling works better for two-way set-associative caches because they have fewer conflict misses than direct-mapped caches [8]. A high rate of conflict misses to a few sets can make those sets poor predictors of overall behavior.

Finally, in practical applications of set sampling, we want to estimate the error of an MPI estimate, using only the information contained within the sample (i.e., not using knowledge


we do this by calculating $90 \%$ confidence intervals, assuming 
TABLE IV

Set Sampling Precision for Direct MapPed

\begin{tabular}{|c|c|c|c|c|c|c|}
\hline \multirow{2}{*}{ Trace } & \multirow{2}{*}{ Size } & \multirow{2}{*}{$M P I_{\text {true }} \times 1000$} & \multicolumn{2}{|c|}{$1 / \overline{16}$ of Sets } & \multicolumn{2}{|c|}{$1 / 64$ of Sets } \\
\hline & & & $\leq \pm 10 \%$ & $\mathrm{CV}$ & $\leq \pm 10 \%$ & $\mathrm{CV}$ \\
\hline \multirow{3}{*}{ MulıI } & $1 \mathrm{M}$ & 1.55 & $16 / 16$ & $4.3 \%$ & N/A & N/A \\
\hline & $4 \mathrm{M}$ & 0.70 & $16 / 16$ & $2.3 \%$ & $62 / 64$ & $4.8 \%$ \\
\hline & $16 \mathrm{M}$ & 0.33 & $16 / 16$ & $1.6 \%$ & $64 / 64$ & $2.7 \%$ \\
\hline \multirow{3}{*}{ Mult!.2 } & $1 \mathrm{M}$ & 1.45 & $16 / 16$ & $2.9 \%$ & N/A & N/A \\
\hline & $4 \mathrm{M}$ & 0.69 & $16 / 16$ & $1.9 \%$ & $62 / 64$ & $4.1 \%$ \\
\hline & $16 \mathrm{M}$ & 0.32 & $16 / 16$ & $1.5 \%$ & $63 / 64$ & $3.2 \%$ \\
\hline \multirow{3}{*}{ Mult2 } & $\mathrm{IM}$ & 1.24 & $16 / 16$ & $3 . \overline{4} \%$ & $\overline{\mathbf{N} / \mathrm{A}}$ & N/A \\
\hline & $4 \mathrm{M}$ & 0.61 & $16 / 16$ & $1.9 \%$ & $64 / 64$ & $2.9 \%$ \\
\hline & $16 \mathrm{M}$ & 0.26 & $16 / 16$ & $2.3 \%$ & $64 / 64$ & $3.3 \%$ \\
\hline \multirow{3}{*}{ Mult 2.2} & $\mathrm{IM}$ & 1.18 & $16 / 16$ & $2.7 \%$ & $\overline{\mathrm{N} / \mathrm{A}}$ & N/A \\
\hline & $4 \mathrm{M}$ & 0.59 & $16 / 16$ & $1.3 \%$ & $64 / 64$ & $2.5 \%$ \\
\hline & $16 \mathrm{M}$ & 0.27 & $16 / 16$ & $1.8 \%$ & $64 / 64$ & $3.4 \%$ \\
\hline \multirow{3}{*}{ Tr } & $\mathrm{IM}$ & 2.63 & $16 / 16$ & $1.9 \%$ & $\mathrm{~N} / \mathrm{A}$ & $\mathrm{N} / \mathrm{A}$ \\
\hline & $4 \mathrm{M}$ & 1.88 & $16 / 16$ & $0.6 \%$ & $64 / 64$ & $2.1 \%$ \\
\hline & $16 \mathrm{M}$ & 1.03 & $16 / 16$ & $0.6 \%$ & $64 / 64$ & $2.0 \%$ \\
\hline \multirow{3}{*}{ Sor } & $1 \mathrm{M}$ & 14.77 & $16 / 16$ & $0.4 \%$ & N/A & N/A \\
\hline & $4 \mathrm{M}$ & 7.54 & $16 / 16$ & $0.3 \%$ & $64 / 64$ & $0.7 \%$ \\
\hline & $16 \mathrm{M}$ & 1.97 & $16 / 16$ & $0.0 \%$ & $64 / 64$ & $0.1 \%$ \\
\hline \multirow{3}{*}{ Tree } & $1 \mathrm{M}$ & 2.16 & $15 / 16$ & $5 . \overline{6 \%}$ & $\mathrm{~N} / \mathrm{A}$ & N/A \\
\hline & $4 \mathrm{M}$ & 0.59 & $14 / 16$ & $6.8 \% \dagger$ & $6 / 64$ & $13.6 \%+$ \\
\hline & $16 \mathrm{M}$ & 0.30 & $15 / 16$ & $4.1 \%$ & $61 / 64$ & $6.5 \%$ \\
\hline \multirow{3}{*}{ Lin } & $1 \mathrm{M}$ & 1.16 & $16 / 16$ & $3.3 \%$ & N/A & N/A \\
\hline & $4 \mathrm{M}$ & 0.09 & $14 / 16$ & $7.6 \% \dagger$ & $54 / 64$ & $15.0 \% \dagger$ \\
\hline & $16 \mathrm{M}$ & 0.02 & $16 / 16$ & $0.3 \%$ & $64 / 64$ & $0.5 \%$ \\
\hline
\end{tabular}

For direct-mapped caches, this table shows the actual MPI of the full trace MPI $_{1 \text { rue }}$, the fraction of set samples with less than or equal to $\pm 10 \%$ relative error and the coefficient of variation of the set-sampling MPI estimates, calculated using (1). We construct samples with the constant-bits method. Samples for $1 / 16$ and $1 / 64$ hold bits $11-8$ and $12-7$ constant, respectively. Some entries marked "N/A" are not available, because the PID hashing overlapped with the constant bits. Except where marked with a dagger $(\dagger)$, at least $90 \%$ of the samples ( $\geq 15$ of 16 or $\geq 58$ of 64 ) have relative errors of less than or equal to $\pm 10 \%$.

TABLE $\mathrm{V}$

SET-SAMPling ERror Prediction

\begin{tabular}{|c|c|cc|cc|}
\hline \multicolumn{5}{|c|}{$90 \%$ Confidence Intervals that Contain $M P I_{\text {rrue }}$} \\
\hline \hline Trace & Normal? & \multicolumn{2}{|c|}{$1 / 16$ of Sets } & \multicolumn{2}{|c|}{$1 / 64$ of Sets } \\
& & fraction & percent & fraction & percent \\
\hline Mult1 & yes & $16 / 16$ & $100 \%$ & $61 / 64$ & $95 \%$ \\
Mult1.2 & yes & $16 / 16$ & $100 \%$ & $60 / 64$ & $94 \%$ \\
Mult2 & yes & $15 / 16$ & $94 \%$ & $61 / 64$ & $95 \%$ \\
Mult2.2 & yes & $16 / 16$ & $100 \%$ & $63 / 64$ & $98 \%$ \\
Tv & no & $16 / 16$ & $100 \%$ & $51 / 64$ & $78 \%$ \\
Sor & yes & $16 / 16$ & $100 \%$ & $64 / 64$ & $100 \%$ \\
Tree & no & $12 / 16$ & $75 \%$ & $47 / 64$ & $73 \%$ \\
Lin & no & $16 / 16$ & $100 \%$ & $62 / 64$ & $97 \%$ \\
\hline
\end{tabular}

For a 4-megabyte direct-mapped secondary cache and various traces and fraction of sets, this table gives the fraction and percent of $90 \%$ confidence

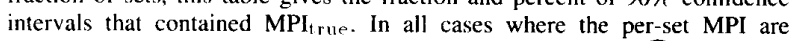
normal, $90 \%$ confidence intervals usefully estimate how far $\widehat{\mathrm{MPI}} \_$is likely to be from MPI $_{\text {true. }}$.

a) random samples and b) that our estimate of the mean is normally distributed. Since variance of observations within our systematic samples is often greater than variance of the population, assumption a) will tend make our confidence intervals larger than necessary. For finite populations, assumption b) will generally hold if the underlying population is not highly skewed [5, Section 2.11].

We empirically studied the usefulness of confidence intervals two ways. First, we tested the validity of assumption b) using normal scores plots (not shown) for sets of 4 megabyte direct-mapped caches [14, p. 172]. Results show that assumption b) is valid for the four multiprogramming traces (Mult1, Mult1.2, Mult2, and Mult2.2) and Sor, but not for Tv, Tree, and Lin. Tree and Lin both have several "hot sets," and these outliers significantly skew their distributions. This suggests that confidence intervals for uniprogrammed traces should not be considered meaningful without additional evidence. Second, we examined how often the $90 \%$ confidence intervals actually included the true mean. Table $\mathrm{V}$ displays data for constant-bits set samples and a 4-megabyte direct-mapped cache. Results show that the true mean lies within the $90 \%$ confidence intervals of at least $90 \%$ of the samples for all traces where the normal approximation appears valid.

\section{Advantages and Disadvantages of Set Sampling}

The most important advantage of set sampling is that, for our simulations, it meets the $10 \%$ sampling goal (Definition 1). Especially for the multiprogrammed traces, a set sample automatically includes references from many execution phases, so an individual sample can accurately characterize the MPI of a full trace, including its temporal variability. The reduced trace data requirements of set sampling allow for simulation of longer traces, and therefore more algorithmic phases, in a smaller amount of time. Besides the data reduction, set sampling also reduces the memory required to simulate a cache. A set sample containing $1 / 16$ of the full trace needs to simulate only $1 / 16$ of the sets.

Set sampling does have its limitations. Even with the constant bits method, the full trace must be retained if one wishes to study caches that do not index with the constant bits. Furthermore, set sampling may not accurately model caches whose performance is affected by interactions between references to different sets. The effectiveness of a prefetch into one set, for example, may depend on how many references are made to other sets before the prefetched block is first used. Similarly, the performance of a cache with a write buffer may be affected by how often the write buffer fills up due to a burst of writes to many sets.

\section{Time SAMPLing}

The alternative to set sampling is time sampling. Here an observation is the MPI of a sequence of time-contiguous references and is called an interval. Section IV-A discusses determining the MPI for a sample, while Section IV-B examines using a sample to estimate MPI for the full trace.

\section{A. Reducing Cold-Start Bias in Time Samples}

To significantly reduce trace storage and simulation time, we must estimate the true MPI for an interval without knowledge of initial cache state, i.e., the cache state at the beginning of the interval. This problem is simply the well-known cold-start problem applied to each interval [6].

The cold-start problem is a key difficulty for time sampling. Sampling theory assumes that a sample is collection of observations, where each observation gives the true value for some member of the population. Set sampling meets this assumption, because computing the true MPI of a set, given all references to the set, is straightforward. Due to the cold-start problem, however, statistics for time sampling are calculated with estimates of the MPI of each interval, rather than the true values of each interval. Any bias in the interval estimates will. of course, remain in all statistics, including the sample mean.

We compare how well the five techniques described in Table VI mitigate the cold-start problem in multi-megabyte caches. 
TABLE VI

Techingues for Mitigating Cold-Start

\begin{tabular}{|c|c|}
\hline Technique & Description \\
\hline COLD & $\begin{array}{l}\text { COLD assumes that the initial cache state is empty. While this assumption does not af- } \\
\text { fect misses to full sets or hits to any sel, it causes COLD to overestimate MPI, hocausse } \\
\text { references that appear to miss to (partially) empty sets may or may not be misses when } \\
\text { simulated with the (true) initial cache state. These potential misses are often called cold- } \\
\text { stan misses [EASF78]. }\end{array}$ \\
\hline HALF & $\begin{array}{l}\text { HALF uses the first half of the instructions in an interval to (partially) initialize the cache. } \\
\text { and estimates MPI with the remaining instructions. }\end{array}$ \\
\hline PRIME & $\begin{array}{l}\text { PRIME estimates MPI with references to "initialized" sets. A set in a direct-mapped } \\
\text { cache is initialized once it is filled [STON90], while a set in a set-associalive cache is inj- } \\
\text { tialized after it is filled and a non-most-recently-used block has been referenced } \\
\text { [LAP188]. }\end{array}$ \\
\hline STITCH & $\begin{array}{l}\text { STITCH approximates the cache state at the beginning of an interval with the cache state } \\
\text { at the end of the previous interval [AGHH88]. Thus one creates a trace for a sample by } \\
\text { stitching it's intervals together. }\end{array}$ \\
\hline INITMR & $\begin{array}{l}\text { Like COLD, INTTMR simulates an interval beginning with an empty initial cache stale. } \\
\text { Instead of assuming that all cold-start misses miss, however, NITMR uses Wood et al.'s } \\
\hat{\mu}_{\text {split to estimate the fraction of cold-start misses that would have missed if the initial }} \\
\text { cacte state was known [WoHK91]. The estimate is based on (1) the fraction of time that } \\
\text { a cache block frame holds a black that will not be referenced before it is replaced, and } \\
\text { (2) the fraction of the cache loaded during the cold-start simulation of an interval. When } \\
\text { we could not estimate (1) with the references in an interval, we assume it to be } 0.7 \text { (based } \\
\text { on the data in Table } 2 \text { of [WoHK91]). }\end{array}$ \\
\hline
\end{tabular}

We will find that none of the five effectively reduce cold-start bias with short intervals (e.g., $<10$ million instructions for 1-megabyte caches).

For a particular trace and cache, we evaluate a cold-start technique as follows. We select the number of instructions in an interval, called the interval length, and collect a systematic sample $S$ of size $n=30$ intervals spaced equally in the trace. (We chose 30 , because it is a commonly-used sample size [14].) We use the cold-start technique to estimate the MPI for each interval, $\widehat{\mathrm{mpi}}_{i}$, and calculate an MPI estimate for sample S with:

$$
\widehat{\mathrm{MPI}}_{S}=\frac{1}{n} \sum_{i=1}^{n} \widehat{\mathrm{mpi}}_{i}
$$

Since with time sampling each interval has the same number of instructions, it is meaningful to compute $\widehat{\mathrm{MPI}}_{S}$ with the arithmetic mean of the $\widehat{\mathrm{mpi}}_{i}$ 's.

Since we have the full trace, we can simulate each interval with its initial cache state to determine the interval's true MPI, mpi $i_{i}$, and calculate the true MPI for the sample, MPI with $\frac{1}{n} \sum_{i=1}^{n} \mathrm{mpi}_{i}$. We evaluate how well a technique reduces cold-start bias in a sample $\mathbf{S}$ with $^{3}$ :

$$
\operatorname{BIAS}_{S}=\frac{\widehat{\mathrm{MPI}}_{S}-\mathrm{MPI}_{S}}{\mathrm{MPI}_{S}} .
$$

It is important to distinguish $\mathrm{MPI}_{\text {true, }} \mathrm{MPI}_{S}$, and $\widehat{\mathrm{MPI}}_{S}$. mpi $_{\text {true }}$ is misses per instruction for all references in the trace (as if all references in the trace are simulated). $\mathrm{MPI}_{S}$ is the misses per instruction of references in the observations of sample $\mathbf{S}$, given each observation starts with its true initial cache state (as if all references since the last observation had been simulated without recording whether they hit or missed). $\widehat{\mathrm{MPI}}_{S}$ is the misses per instruction of references in the observations of sample $\mathbf{S}$, given each observation starts

\footnotetext{
${ }^{3}$ We calculate BIAS $_{S}$ for PRIME with the secondary cache's local miss ratio rather than MPI, because counting the number of instructions is not straightforward when some sets are initialized but others are not. Since BLAS is a relative error, we expect that calculating it with local miss ratio will be comparable to calculating it with MPI.
}

TABLE VII

\begin{tabular}{|c|c|c|c|c|c|c|c|}
\hline Trace & $\begin{array}{l}\text { Cache } \\
\text { Size }\end{array}$ & $M P I_{S} \times 1000$ & COLD & HALF & PRIME & STITCH & INITMR \\
\hline Mult 1 & $\begin{array}{r}1 \mathrm{M} \\
4 \mathrm{M} \\
16 \mathrm{M}\end{array}$ & $\begin{array}{l}1.45 \\
0.62 \\
0.28\end{array}$ & $\begin{array}{r}+18 \% \\
+77 \% \\
+233 \%\end{array}$ & $\begin{array}{r}+5 \% \\
+27 \% \\
+114 \%\end{array}$ & $\begin{array}{l}-18 \% \\
-50 \% \\
-80 \%\end{array}$ & $\begin{array}{r}+23 \% \\
+52 \% \\
+131 \%\end{array}$ & $\begin{array}{l}+0 \% \\
-11 \% \\
-12 \%\end{array}$ \\
\hline Mult1.2 & $\begin{array}{r}1 \mathrm{M} \\
4 \mathrm{M} \\
16 \mathrm{M}\end{array}$ & $\begin{array}{l}1.57 \\
0.77 \\
0.37\end{array}$ & $\begin{array}{r}+16 \% \\
+66 \% \\
+200 \%\end{array}$ & $\begin{array}{r}+2 \% \\
+25 \% \\
+103 \% \\
\end{array}$ & $\begin{array}{l}-18 \% \\
-51 \% \\
-80 \%\end{array}$ & $\begin{array}{r}+2 \% \\
+27 \% \\
+90 \%\end{array}$ & $\begin{array}{l}+2 \% \\
-5 \% \\
-3 \%\end{array}$ \\
\hline Mult2 & $\begin{array}{r}1 \mathrm{M} \\
4 \mathrm{M} \\
16 \mathrm{M}\end{array}$ & $\begin{array}{l}1.21 \\
0.60 \\
0.25\end{array}$ & $\begin{array}{r}+18 \% \\
+70 \% \\
+264 \%\end{array}$ & $\begin{array}{r}+2 \% \\
+31 \% \\
+168 \% \\
\end{array}$ & $\begin{array}{l}-26 \% \\
-62 \% \\
-85 \%\end{array}$ & $\begin{array}{r}+23 \% \\
+53 \% \\
+147 \%\end{array}$ & $\begin{array}{r}-3 \% \\
-24 \% \\
-9 \%\end{array}$ \\
\hline Mulı.2.2 & $\begin{array}{r}1 \mathrm{M} \\
4 \mathrm{M} \\
16 \mathrm{M} \\
\end{array}$ & $\begin{array}{l}.18 \\
0.62 \\
0.29 \\
\end{array}$ & $\begin{array}{r}+19 \% \\
+71 \% \\
+233 \% \\
\end{array}$ & $\begin{array}{r}+15 \% \\
+50 \% \\
+180 \% \\
\end{array}$ & $\begin{array}{l}-27 \% \\
-61 \% \\
-84 \% \\
\end{array}$ & $\begin{array}{r}+29 \% \\
+56 \% \\
+141 \% \\
\end{array}$ & $\begin{array}{r}-1 \% \\
-13 \% \\
-3 \% \\
\end{array}$ \\
\hline $\mathrm{Tv}$ & $\begin{array}{r}1 \mathrm{M} \\
4 \mathrm{M} \\
16 \mathrm{M}\end{array}$ & $\begin{array}{l}2.55 \\
1.76 \\
0.95\end{array}$ & $\begin{array}{r}+4 \% \\
+15 \% \\
+79 \%\end{array}$ & $\begin{array}{r}-0 \% \\
+9 \% \\
+61 \%\end{array}$ & $\begin{array}{l}-33 \% \\
-56 \% \\
-76 \%\end{array}$ & $\begin{array}{l}+32 \% \\
+37 \% \\
+71 \%\end{array}$ & $\begin{array}{r}-2 \% \\
-4 \% \\
+37 \%\end{array}$ \\
\hline Sor & $\begin{array}{r}1 M \\
4 M \\
16 M \\
\end{array}$ & $\begin{array}{r}15.68 \\
8.08 \\
2.00 \\
\end{array}$ & $\begin{array}{r}+0 \% \\
+18 \% \\
+190 \% \\
\end{array}$ & $\begin{array}{r}-0 \% \\
+2 \% \\
+60 \% \\
\end{array}$ & $\begin{array}{r}-5 \% \\
-18 \% \\
-76 \% \\
\end{array}$ & $\begin{array}{r}-11 \% \\
-8 \% \\
-8 \% \\
\end{array}$ & $\begin{array}{r}0 \% \\
+6 \% \\
+114 \% \\
\end{array}$ \\
\hline Tree & $\begin{array}{r}1 \mathrm{M} \\
4 \mathrm{M} \\
16 \mathrm{M}\end{array}$ & $\begin{array}{l}2.00 \\
0.51 \\
0.30\end{array}$ & $\begin{array}{r}+13 \% \\
+107 \% \\
+217 \%\end{array}$ & $\begin{array}{r}-0 \% \\
+8 \% \\
+35 \%\end{array}$ & $\begin{array}{l}-10 \% \\
-50 \% \\
-77 \%\end{array}$ & $\begin{array}{l}+29 \% \\
+43 \% \\
+69 \%\end{array}$ & $\begin{array}{r}-1 \% \\
+24 \% \\
+18 \%\end{array}$ \\
\hline Lin & $\begin{array}{c}1 \mathrm{MM} \\
4 \mathrm{M} \\
16 \mathrm{M}\end{array}$ & $\begin{array}{l}0.75 \\
0.06 \\
0.01\end{array}$ & $\begin{array}{r}+20 \% \\
+1113 \% \\
+4648 \%\end{array}$ & $\begin{array}{r}+7 \% \\
+535 \% \\
+2248 \%\end{array}$ & $\begin{array}{l}-29 \% \\
-62 \% \\
\ldots \%\end{array}$ & $\begin{array}{r}-0 \% \\
+217 \% \\
+873 \%\end{array}$ & $\begin{array}{r}+16 \% \\
+903 \% \\
+1037 \%\end{array}$ \\
\hline
\end{tabular}

Bias of Cold-Start Technigues with DiRect-Mapped CaChes

This table displays BIAS $_{S}$ for five cold-start techniques, eight traces, an interval length of 10 million instructions, and three direct-mapped cache sizes $(1,4$, and 16 megabytes)

with an initial cache state approximated by some cold-start technique.

Since BIAS $_{S}$ compares $\widehat{\mathrm{MPI}}_{S}$ with $\mathrm{MPI}_{S}$, rather than MPI $_{\text {true }}$, it measures cold-start bias in the sample, not how well the sample predicts MPI $_{\text {true }}$. We consider how well time samples predict MPI $_{\text {true }}$ in Section IV-B.

We evaluate BIAS $_{S}$ for five cold-start techniques, eight traces, four interval lengths (100 thousand, 1 million, 10 million, and 100 million instructions), three cache sizes (1, 4, and 16 megabytes) and two associativities (direct-mapped and four-way). Since space precludes us from displaying 192 cases for each cold-start technique, we present several subsets of the data.

For a 10-million-instruction interval length, Table VII displays BIAS $_{S}$ for direct-mapped caches, while Table XI in Appendix $C$ gives similar data for four-way set-associative caches. The data show several trends. First, most BIAS $_{S}$ 's are large, especially for caches larger than one megabyte. This suggests that intervals longer than many previously published traces are needed to effectively reduce cold-start bias for multi-megabyte caches. Second, COLD, HALF and STITCH tend to overestimate MPI $_{S}$. COLD does so because it assumes that all cold-start misses miss. Similarly, HALF tends to overestimate $\mathrm{MPI}_{S}$ when the first half of the trace does not sufficiently fill the cache. HALF can underestimate the sample's MPI, however, when the second half of most of a sample's intervals have a lower MPI than the whole of each interval. We believe STITCH overestimates $\mathrm{MPI}_{S}$, because (due to temporal locality) references are less likely to miss when simulated with an interval's true initial state than with the final state from the previous interval [24]. Third, PRIME underestimates $\mathrm{MPI}_{S}$ for direct-mapped caches. PRIME calculates $\mathrm{MPI}_{S}$ by effectively assuming that coldstart misses are as likely to miss as any other reference. Wood et al. [25] have shown, however, that this assumption is false, and that cold-start misses are much more likely to miss than 
TABLE VIII

SCoring of Different Cold-STart Techniques

\begin{tabular}{|c|c|c|c|c|c|c|c|c|c|c|c|}
\hline \multirow{2}{*}{$\begin{array}{c}\text { Cache } \\
\text { Size }\end{array}$} & \multirow{2}{*}{$\begin{array}{l}\text { Interval } \\
\text { Length } \\
\text { (Mill) }\end{array}$} & \multicolumn{2}{|c|}{ COLD } & \multicolumn{2}{|c|}{ HALF } & \multicolumn{2}{|c|}{ PRIME } & \multicolumn{2}{|c|}{ STITCH } & \multicolumn{2}{|c|}{ INITMR } \\
\hline & & $10 \%$ & Win & $10 \%$ & Win & $10 \%$ & Win & $10 \%$ & Win & $10 \%$ & Win \\
\hline \multirow{4}{*}{$\mathrm{IM}$} & 0.1 & 2 & 0 & 2 & 5 & 0 & 2 & 0 & 0 & 0 & 9 \\
\hline & 1 & 2 & 1 & 4 & 4 & 3 & 5 & I & 2 & 3 & 5 \\
\hline & 10 & 4 & 2 & 15 & 13 & 7 & 1 & 4 & 3 & 12 & 8 \\
\hline & 100 & 16 & 5 & 16 & 7 & 16 & 6 & 6 & 2 & 16 & 12 \\
\hline \multirow{4}{*}{$4 \mathrm{M}$} & 0.1 & 0 & 0 & 0 & 0 & 0 & 1 & 1 & 2 & 1 & 13 \\
\hline & 1 & 0 & 0 & 0 & 1 & 1 & 2 & 2 & 1 & 4 & 13 \\
\hline & 10 & 1 & 0 & 6 & 6 & 0 & 1 & 2 & 1 & 10 & 8 \\
\hline & 100 & 7 & 1 & 14 & 4 & 3 & 2 & 5 & 3 & 12 & 7 \\
\hline \multirow{4}{*}{$16 \mathrm{M}$} & 0.1 & 0 & 0 & 0 & 0 & $\overline{0}$ & 0 & 0 & 0 & 0 & 16 \\
\hline & I & $\begin{array}{l}0 \\
0\end{array}$ & 0 & 0 & 0 & 0 & 0 & 1 & 2 & 0 & 14 \\
\hline & 10 & 0 & 0 & 0 & 0 & 0 & 1 & 2 & 4 & 6 & 11 \\
\hline & 100 & 0 & 0 & 3 & 2 & 1 & 0 & 5 & 9 & 5 & 5 \\
\hline \multirow{4}{*}{ All } & 0.1 & 2 & 0 & 2 & 5 & 0 & 3 & 1 & 2 & 1 & 38 \\
\hline & 1 & 2 & I & 4 & 5 & 4 & 7 & 5 & 5 & 7 & 32 \\
\hline & 10 & 5 & 2 & 21 & 19 & 7 & 3 & 8 & 8 & 28 & 27 \\
\hline & 100 & 23 & 6 & 33 & 13 & 20 & 8 & 16 & 14 & 33 & 24 \\
\hline All & All & 32 & 9 & 60 & 42 & 31 & 21 & 29 & 29 & 69 & 121 \\
\hline
\end{tabular}

This table displays scores of the cold-start techniques for 192 cases: the eight traces, four interval lengths (100 thousand, 1 million, 10 million, and 100 million instructions), three cache sizes $(1,4$, and 16 megabytes) and two associativities (direct-mapped and four-way). We award a point in the $10 \%$ category if $-10 \% \leq$ BIAS $S \leq 10 \%$ and award one in the "Win" category for the cold-start technique closest to being unbiased $\left(\log \left|\mathrm{BIAS}_{s}\right|\right.$ closest to zero). Multiple points are awarded in the case of ties.

randomly-chosen references. PRIME is more accurate for fourway set-associative caches, where the heuristic of ignoring initial references to a most-recently-referenced block mitigates the underestimation. Fourth, INITMR did not consistently underestimate or overestimate $\mathrm{MPI}_{S}$. Finally, the large biases for the Lin trace with 4- and 16-megabyte caches are probably not important, because Lin's true MPI's are so small.

Table VIII addresses which cold-start technique is best for these traces and caches. For each the five cold-start techniques, we compute BIAS $_{S}$ for all 192 cases. We award a point in the " $10 \%$ " category for biases less than $\pm 10 \%$ and award one in the "Win" category for the cold-start technique closest to being unbiased. Multiple points are awarded in the case of ties. The final row of Table VIII gives totals. HALF and INITMR have twice the " $10 \%$ " score of the other approaches, while INITMR has more "Wins" than all the other approaches combined. While HALF performs well in many cases, INITMR performs best overall. While results for other traces and cache could differ, the theory behind INITMR [25] and this experimental evidence strongly support INITMR. For these reasons, we will use it in the rest of this paper.

Table IX illustrates how well INITMR performs with three direct-mapped caches (1, 4, and 16 megabytes) and all four interval lengths $(100,000,1,000,000,10,000,000$, and 100,000,000 instructions). As expected, it reduces bias more effectively as the interval lengths get longer or cache size gets smaller, because cold-start becomes less dominant. The most striking aspect of this data is that INITMR, the best method, still performs terribly for intervals containing 100,000 and $1,000,000$ instructions. This should not be not surprising, since the number of block frames in the caches (e.g., 8192 for 1-megabyte caches) far exceeds the number of true misses in these intervals (e.g., 1550 equals $1,000,000$ instructions times a 0.00155 MPI for Mult1). Furthermore, it appears that INITMR does not adequately mitigate coldstart bias unless interval lengths are, at least, 10 million instructions for 1-megabyte caches, 100 million instructions
TABLE IX

BIAS $_{S}$ OF INITMR TIME-SAMPLE MPI EstimateS

\begin{tabular}{|c|c|c|c|c|c|c|}
\hline \multirow{2}{*}{ Trace } & \multirow{2}{*}{$\begin{array}{c}\text { Cache } \\
\text { Size }\end{array}$} & \multirow{2}{*}{$M P I_{\text {true }} \times 1000$} & \multicolumn{4}{|c|}{ Interval Length (Millions of Instructions) } \\
\hline & & & 0.1 & 1 & 10 & 100 \\
\hline \multirow[t]{3}{*}{ Mult1 } & $1 \mathrm{M}$ & 1.55 & $86 \%$ & $47 \%$ & $0 \%{ }^{*}$ & $0 \% *$ \\
\hline & $4 \mathrm{M}$ & 0.70 & $156 \%$ & $120 \%$ & $-11 \%$ & $-3 \% *$ \\
\hline & $16 \mathrm{M}$ & 0.33 & $281 \%$ & $335 \%$ & $-12 \%$ & $-17 \%$ \\
\hline \multirow[t]{3}{*}{ Mult1.2 } & $1 \mathrm{M}$ & 1.45 & $103 \%$ & $21 \%$ & $2 \% *$ & $0 \% *$ \\
\hline & $4 \mathrm{M}$ & 0.69 & $123 \%$ & $63 \%$ & $-5 \%$ & $-2 \% *$ \\
\hline & $16 \mathrm{M}$ & 0.32 & $400 \%$ & $100 \%$ & $-3 \%$ & $-17 \%$ \\
\hline \multirow[t]{3}{*}{ Mult2 } & IM & 1.24 & $49 \%$ & $20 \%$ & $-3 \%^{*}$ & $0 \% *$ \\
\hline & $4 \mathrm{M}$ & 0.61 & $48 \%$ & $39 \%$ & $-24 \%$ & $0 \% *$ \\
\hline & $16 \mathrm{M}$ & 0.26 & $212 \%$ & $146 \%$ & $-9 \%$ & $-3 \%$ \\
\hline \multirow[t]{3}{*}{ Mult 2.2} & $1 \mathrm{M}$ & 1.18 & $127 \%$ & $24 \%$ & $-1 \% *$ & $0 \% *$ \\
\hline & $4 \mathrm{M}$ & 0.59 & $127 \%$ & $60 \%$ & $.13 \%$ & $0 \% *$ \\
\hline & $16 \mathrm{M}$ & 0.27 & $170 \%$ & $106 \%$ & $-3 \%$ & $8 \%$ \\
\hline \multirow[t]{3}{*}{$\mathrm{Tv}$} & $1 \mathrm{M}$ & 2.63 & $36 \%$ & $-10 \%$ & $-2 \% *$ & $0 \% *$ \\
\hline & $4 \mathrm{M}$ & 1.88 & $34 \%$ & $-9 \%$ & $-4 \%$ & $0 \% *$ \\
\hline & $16 \mathrm{M}$ & 1.03 & $145 \%$ & $39 \%$ & $37 \%$ & $12 \%$ \\
\hline \multirow[t]{3}{*}{ Sor } & $1 \mathrm{M}$ & 14.77 & $-41 \%$ & $-3 \% *$ & $0 \% *$ & $0 \% *$ \\
\hline & $4 M$ & 7.54 & $-27 \%$ & $44 \%$ & $6 \% *$ & $0 \% *$ \\
\hline & $16 \mathrm{M}$ & 1.97 & $83 \%$ & $386 \%$ & $114 \%$ & $-2 \% *$ \\
\hline \multirow[t]{3}{*}{ Tree } & $\mathrm{IM}$ & 2.16 & $249 \%$ & $36 \%$ & $-1 \%{ }^{*}$ & $0 \% *$ \\
\hline & $4 M$ & 0.59 & $1407 \%$ & $121 \%$ & $24 \%$ & $-7 \% *$ \\
\hline & $16 \mathrm{M}$ & 0.30 & $796 \%$ & $198 \%$ & $18 \%$ & $-37 \%$ \\
\hline \multirow[t]{3}{*}{$\operatorname{Lin}$} & $1 \mathrm{M}$ & 1.16 & $-30 \%$ & $-14 \%$ & $16 \%$ & $1 \% *$ \\
\hline & $4 \mathrm{M}$ & 0.09 & $1437 \%$ & $946 \%$ & $903 \%$ & $113 \%$ \\
\hline & $16 \mathrm{M}$ & 0.02 & $2567 \%$ & $1318 \%$ & $1037 \%$ & $176 \%$ \\
\hline
\end{tabular}

This table displays $B I+S_{S}$ for INITMR with eight traces, four interval lengths, three direct-mapped cache sizes $(1,2$, and 16 megabytes). We mark entries with an asterisk ("**) if , on average, interval lengths are sufficient to a) fill at least haf the cache and b) there are at least as many misses to full sets as cold-start misses.

for 4-megabyte caches, and more than 100 million instructions for 16-megabyte caches. These results are consistent with the rule-of-thumb that trace length should be increased by a factor of eight each time the cache size quadruples [22].

As Table IX also illustrates, however, we can determine when INITMR is likely to perform well. We marked each entry in the table with an asterisk ("**) if, on average, the interval length was sufficient to a) fill at least half the cache and b) there were at least as many misses to full sets as cold-start misses. All values BIAS $_{S}$ marked with an asterisk are less than $\pm 10 \%$. Nevertheless, they imply that for multi-megabyte caches each interval should contain more instructions than have previously been present in many "full" traces.

\section{B. What Fraction of the Full Trace is Needed?}

This section examines how accurately time samples estimate MPI $_{\text {true }}$, the MPI of the full trace. We estimate the MPI of a sample $\mathbf{S}, \widehat{\mathrm{MPI}}_{S}$, with the arithmetic mean of MPI estimates for each interval in the sample, where we use INITMR to reduce (but regrettably not eliminate) the cold-start bias of each interval.

Figure 4(a) illustrates how we summarize the data. (We use a graphical display here instead of coefficient of variation, because we believe it provides more insight. We did not use a graphical display with set sampling, because we did not have enough samples to smooth the data.) For the Mult 1.2 traces and a 4-megabyte direct-mapped cache, it plots $\widehat{\mathrm{MPI}}_{S} / \mathrm{MPI}_{\text {true }}$ on the logarithmic $y$-axis and the fraction of the full trace contained in the sample on the logarithmic $x$-axis. Consider the cone at the far left. We use 30001 -million-instruction intervals to calculate its shape. The left edge, near 0.00025 , gives the fraction of the trace used in a sample of one interval. We determine the end-points of the left edge with the empirical distribution of $\widehat{\mathrm{MPI}}_{S}$ for single-interval samples. The upper end-point gives the 95th percentile, while the lower gives the 5 th 




(a)

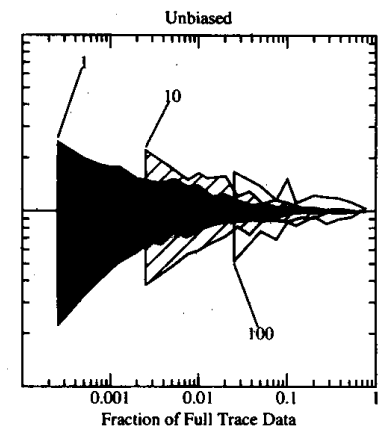

(b)
Fig. 4. Cones for time sampling with Mult1.2. (a) cones for $\widehat{\mathrm{MPI}}_{S}$. (b) Cones for MPI $S_{S}$ (no hat). This figure displays cones for $\widehat{\mathrm{MPI}}_{S}$ (left) and MPI ${ }_{S}$ (right) for the Mult1.2 trace and a 4-megabyte direct-mapped cache. For an interval length and sample size (whose product gives the fraction of the trace used) the height of a cone displays the range of the middle $90 \%$ of estimates from many samples. Estimates are unbiased only if they are vertically centered on the horizontal line at 1.0. For an interval length of 1 million instructions, for example, all $\widehat{\mathrm{MPI}}_{S}$ displayed here are biased (by cold-start bias not removed

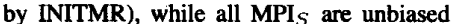

percentile. Thus, the length of the left edge is the range of the middle $90 \%$ of the $\widehat{\mathrm{MPI}}_{S}$ 's. We compute other vertical slices similarly. A vertical line (not shown) in the same cone at 0.01 $(40 \times 0.00025)$, for example, gives the range of the middle $90 \%$ of the $\mathrm{MPI}_{S}$ 's for samples of 40 intervals each. The other two cones are for interval lengths of 10 million instructions (300 intervals) and 100 million instructions ( 30 intervals). The right graph gives similar data for $\mathrm{MPI}_{S}$, where we calculate the MPI of each interval with its true initial cache state.

A time sample would meet the $10 \%$ sampling goal (Definition 1) if (a) the sample's size times the length of each interval were less than $10 \%$ of the trace (e.g., to the left of $x$-axis value 0.1 in Fig. 4(a) and (b) the cone lies between 0.9 and 1.1 (on the $y$-axis). Unfortunately, none of the three cones for Mult1.2 qualify. The cone for 1-million-instruction intervals is narrow enough but biased too far above 1.0, while the cones of 10 million and 100 million instructions are too wide.

We found similar results for the rest of the traces, displayed in Fig. 5(a) and (b) of Appendix C. The cones for the multiprogrammed traces are similar to those of Mult1.2, although Mult2 and Mult2.2 have more cold-start bias. The cones for the single applications, Tree, Tv, Sor, and Lin, are more idiosyncratic, reflecting application-specific behavior. The cones of Sor, for example, are skewed by Sor's behavior of alternating between low and high MPI (with a period of around 300 million instructions [4])

Thus, for these traces and caches (and for direct-mapped and four-way, 1- and 16-megabyte caches [10] time sampling fails to meet the $10 \%$ sampling goal. Furthermore, even if we eliminate cold-start bias, accurate estimates of MPI $_{\text {true }}$ must use hundred of millions of instructions to capture temporal workload variations. With Mult1.2 and a 4-megabyte directmapped cache, Fig. 4(b) shows that $\mathrm{MPI}_{S}$ is within $10 \%$ of $\mathrm{MPI}_{\text {true }}$ (for $90 \%$ of the samples examined) only with samples of 200 intervals of length 1 million instructions, 65 10-millioninstruction intervals, or 20100 -million-instruction intervals. (For much smaller caches, Laha et al. found a sample size of
35 intervals to be sufficient [12].) This is roughly a factor of three decrease in sample size as interval length is multiplied by ten.

Finally, we investigate whether the error in $\widehat{\mathrm{MPI}}_{S}$ can be estimated from information within the sample itself. We calculate $90 \%$ confidence intervals with the same methods as were used for set sampling (Appendix B). These methods, however, provided no information on the magnitude of coldstart bias, because they assume a sample is made up of unbiased observations. Since the cold-start bias (that was not removed by INITMR) is significant in many cases, $90 \%$ confidence intervals for time samples often do not contain MPI $_{\text {true }} 90 \%$ of the time.

Confidence intervals did work in a few cases where samples contained 30 or more intervals and interval lengths were long enough to make cold-start bias negligible [10]. These cases, however, failed to meet the $10 \%$ sampling goal because the samples contained much more than $10 \%$ of the trace. Confidence intervals also worked for $\mathrm{MPI}_{S}$ (whose expected value is MPI $_{\text {true }}$ because it has no cold-start bias), when samples contain at least 30 intervals.

\section{Advantages and Disadvantages of Time Sampling}

The major advantage of time sampling is that it is the only sampling technique available for caches with timing-dependent behavior (e.g., that prefetch or are lockup-free [11]) or shared structures across sets (e.g., write buffers or victim caching [9]). Furthermore, the cold-start techniques for time sampling can be applied to any full-trace simulation, since a "full" trace is just a single, long observation from a system's workload.

However, in these simulations, time sampling fails to meet the $10 \%$ sampling goal for multi-megabyte caches, because it needed long intervals to mitigate cold-start bias and many intervals to capture temporal workload variation. For the cold start techniques we examined, set sampling is more effective than time sampling at estimating the MPI's of our traces with multi-megabyte caches.

\section{CONCLUSION}

A straightforward application of trace-driven simulation to multi-megabyte caches requires very long traces that strain computing resources. Resource demands can be greatly reduced using set sampling or time sampling. Set sampling estimates cache performance using information from a collection of sets, while time sampling uses information from a collection of trace intervals.

This study is the first to apply set sampling and time sampling to multi-megabyte caches, where they are most useful. We use eight billion-reference traces of large workloads that include multiprogramming but not operating system references [4]. Given a trace and cache, we examine how well both techniques predict the misses per instruction (MPI) of the entire trace. We say a sampling method is effective if it meets the $10 \%$ sampling goal: a method meets this goal if, at least $90 \%$ of the time, it estimates the trace's true misses per instruction with $\leq 10 \%$ relative error using $\leq 10 \%$ of the trace. Like most trace-driven simulation studies, we do not formally address how our traces relate to the population of 


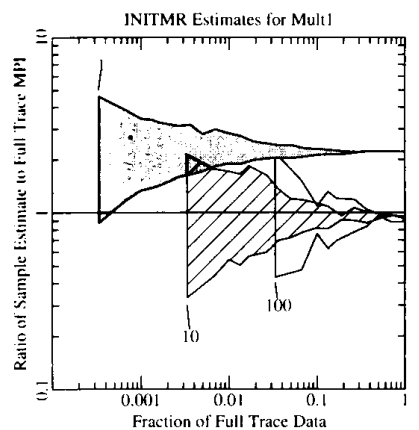

INITMR Estimates for Mult2.2
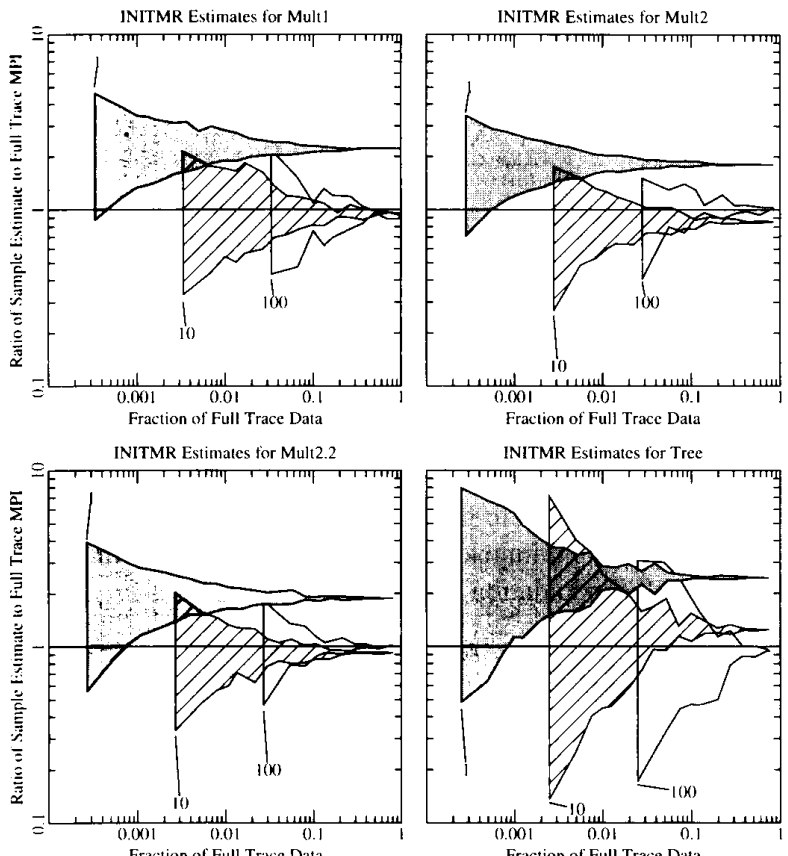

Fraction of Full Trace Dat INITMR Estimates for Tree



Fig. 5(a). Cones for time sampling with Mult1, Mult2, Mult2.2, and Tree. Similar to Fig. 4(a), these figures display cones for $\widehat{M P I}_{S}$ with the Mult 1 , Mult2, Mult2.2, and Tree traces.

all traces. Readers may accept our results (by assuming our traces are representative of their workload) or re-apply our techniques to their traces.

With our traces and caches, we obtained several results for set sampling. First, how we compute MPI is important. We find that it is much less accurate to normalize misses by the instruction fetches to the sampled sets than by the fraction of sampled sets times all instruction fetches. Second, constructing samples from sets that share some common index bit values works well, since such samples can be used to accurately predict the MPI of multiple alternative caches and caches in hierarchies. Third, sets for our multiprogramming traces behave sufficiently close to normal that confidence intervals are meaningful and accurate. Last and most important, for our traces and caches, set sampling meets the $10 \%$ sampling goal.

With our traces and caches, results for time sampling include the following. First, INITMR (Wood et al.'s $\hat{\mu}_{\text {split }}$ [25]) was the most effective technique for reducing cold-start bias, although using half the references in a trace interval to (partially) initialize a cache often performed well. Second, interval lengths must be long to mitigate cold-start bias ( 10 million instructions for 1-megabyte caches, 100 million instructions for 4-megabyte caches, and more than 100 million instructions for 16-megabyte caches). Third and most important, for these traces and caches, time sampling does not meet the $10 \%$ sampling goal: we needed more than $10 \%$ of a trace to get (trace) interval lengths that adequately mitigated cold-start bias and have enough intervals in a sample to make accurate predictions.

Thus, we found that for our traces, set sampling is more effective than time sampling for estimating MPI of the


Fig. 5(b). Cones for time sampling with Tv, Sor, and Lin. Similar to Fig. 4(a), these figures display cones for $\widehat{\mathrm{MPI}}_{s}$ with the Tv, Sor, and Lin traces. Note that Lin uses a different $y$-axis scale.

multi-megabyte caches. There are situations, however, when set sampling is not applicable, such as for caches that have time-dependent behavior (e.g., prefetching) or structures used by many sets (e.g., write buffers). In these cases, researchers must choose between using an entire trace and using time sampling. Since any trace can be considered a time sample of size one, either approach requires care to reduce the effect of cold-start bias.

\section{APPENDIX A \\ SYSTEMATIC SAMPLES}

This appendix introduces systematic samples with a discussion derived from Cochran [5, ch. 8]. We use the notation introduced in Section III for consistency.

The variance of the mean of a random sample of size $n$ from a population of size $s$ is $[8,(2.8)]$ :

$$
\frac{1}{s-1} \sum_{i=1}^{s}\left(\mathrm{mpi}_{i}-\mathrm{MPI}_{\text {true }}\right)^{2} \times \frac{1}{n} \times \frac{(s-n)}{s},
$$

where mpi $_{i}$ is the $i$ th member of the population and MPI true $_{\text {th }}$ is the population mean.

With systematic sampling, a population of size $s$ is systematically divided into $k$ samples of size $n$. By definition, the variance of the mean of a systematic sample, an unbiased estimate of $\mathrm{MPI}_{\text {true }}$, is [8, p. 208]:

$$
\frac{1}{k} \sum_{j=1}^{k}\left(\widehat{\mathrm{MPI}}_{S(j)}-\mathrm{MPI}_{\text {true }}\right)^{2} \text {. }
$$

where $\widehat{\mathrm{MPI}}_{\left.S_{(} j\right)}$ is the mean of the $j$ th systematic sample. 
TABLE $\mathrm{X}$

Set Sampling Precision for 2-Way

\begin{tabular}{|c|c|c|c|c|c|c|}
\hline \multirow{2}{*}{ Trace } & \multirow{2}{*}{ Size } & \multirow{2}{*}{$M P I_{\text {true }} \times 1000$} & \multicolumn{2}{|c|}{$1 / 16$ of Sets } & \multicolumn{2}{|c|}{$1 / 64$ of Sets } \\
\hline & & & $\leq \pm 10 \%$ & $\mathrm{CV}$ & $\leq \pm 10 \%$ & CV \\
\hline \multirow{3}{*}{ Multl } & IM & 1.19 & $16 / 16$ & $2.2 \%$ & N/A & N/A \\
\hline & $4 \mathrm{M}$ & 0.55 & $16 / 16$ & $1.7 \%$ & $64 / 64$ & $3.0 \%$ \\
\hline & $16 \mathrm{M}$ & 0.26 & $16 / 16$ & $1.6 \%$ & $64 / 64$ & $2.3 \%$ \\
\hline \multirow{3}{*}{ Mult1.2 } & $\mathbf{I M}$ & 1.18 & $16 / 16$ & $1.6 \%$ & $\mathbf{N} / \mathbf{A}$ & N/A \\
\hline & $4 \mathrm{M}$ & 0.56 & $16 / 16$ & $1.2 \%$ & $64 / 64$ & $2.2 \%$ \\
\hline & $16 \mathrm{M}$ & 0.28 & $16 / 16$ & $1.3 \%$ & $64 / 64$ & $2.1 \%$ \\
\hline \multirow{3}{*}{ Mult2 } & $1 \mathrm{M}$ & 1.01 & $16 / 16$ & $1.9 \%$ & N/A & N/A \\
\hline & $4 M$ & 0.52 & $16 / 16$ & $1.2 \%$ & $64 / 64$ & $2.0 \%$ \\
\hline & $16 \mathrm{M}$ & 0.24 & $16 / 16$ & $1.9 \%$ & $64 / 64$ & $3.3 \%$ \\
\hline \multirow{3}{*}{ Mult2.2 } & $1 \mathrm{M}$ & 0.98 & $16 / 16$ & $1.8 \%$ & N/A & N/A \\
\hline & $\mathbf{4 M}$ & 0.51 & $16 / 16$ & $1.5 \%$ & $64 / 64$ & $1.9 \%$ \\
\hline & $16 \mathrm{M}$ & 0.22 & $16 / 16$ & $2.1 \%$ & $64 / 64$ & $3.5 \%$ \\
\hline \multirow{3}{*}{$T \mathbf{v}$} & $\mathrm{IM}$ & 2.31 & $16 / 16$ & $0.6 \%$ & $\mathrm{~N} / \mathrm{A}$ & N/A \\
\hline & $4 M$ & 1.76 & $16 / 16$ & $0.3 \%$ & $64 / 64$ & $1.6 \%$ \\
\hline & $16 \mathrm{M}$ & 0.98 & $16 / 16$ & $0.7 \%$ & $64 / 64$ & $1.9 \%$ \\
\hline \multirow{3}{*}{ Sor } & IM & 14.66 & $16 / 16$ & $0.3 \%$ & $\mathrm{~N} / \mathrm{A}$ & N/A \\
\hline & $\mathbf{4 M}$ & 7.76 & $16 / 16$ & $0.2 \%$ & $64 / 64$ & $0.5 \%$ \\
\hline & $16 \mathrm{M}$ & 1.92 & $16 / 16$ & $0.0 \%$ & $64 / 64$ & $0.1 \%$ \\
\hline \multirow{3}{*}{ Tree } & IM & 1.81 & $16 / 16$ & $3.7 \%$ & N/A & N/A \\
\hline & $\mathbf{4 M}$ & 0.49 & $16 / 16$ & $1.5 \%$ & $64 / 64$ & $3.8 \%$ \\
\hline & $16 \mathrm{M}$ & 0.26 & $16 / 16$ & $0.4 \%$ & $64 / 64$ & $1.1 \%$ \\
\hline \multirow{3}{*}{ Lin } & & 1.10 & $16 / 16$ & $2.6 \%$ & N/A & N/A \\
\hline & $4 M$ & 0.06 & $16 / 16$ & $6.0 \%$ & $44 / 64$ & $9.8 \% \dagger$ \\
\hline & $16 \mathrm{M}$ & 0.02 & $16 / 16$ & $0.3 \%$ & $64 / 64$ & $0.5 \%$ \\
\hline
\end{tabular}

This table shows the MPI of the full trace for two-way set-associative caches, the fraction of set samples with less than or equal to $\pm 10 \%$ relative error and the coefficient of variation of the set-sampling MPI estimates, similar to Table IV. Except where marked with a dagger $(\dagger)$, at least $90 \%$ of the samples have relative errors of less than or equal to $\pm 10 \%$.

TABLE XI

Bias of Cold-Start Techniques with Four-Way Set-Associativity

\begin{tabular}{|c|c|c|c|c|c|c|c|}
\hline Trace & $\begin{array}{l}\text { Cache } \\
\text { Size }\end{array}$ & $\mathrm{MPl}_{S} \times 1000$ & COLD & HALF & PRIME & STITCH & INITMR \\
\hline \multirow{3}{*}{ Mult I } & IM & 0.94 & $+21 \%$ & $-5 \%$ & $-6 \%$ & $+36 \%$ & $-11 \%$ \\
\hline & $4 \mathrm{M}$ & 0.44 & $+106 \%$ & $+29 \%$ & $-51 \%$ & $+80 \%$ & $-4 \%$ \\
\hline & $16 \mathrm{M}$ & 0.22 & $+313 \%$ & $+157 \%$ & $.99 \%$ & $+167 \%$ & $-8 \%$ \\
\hline \multirow{3}{*}{ Mult1.2 } & $1 \mathrm{M}$ & 1.20 & $+15 \%$ & $-5 \%$ & $-9 \%$ & $+6 \%$ & $-7 \%$ \\
\hline & $4 \mathrm{M}$ & 0.60 & $+81 \%$ & $+21 \%$ & $-40 \%$ & $+43 \%$ & $+1 \%$ \\
\hline & $\mathrm{i} 6 \mathrm{M}$ & 0.32 & $+232 \%$ & $+118 \%$ & $-57 \%$ & $+104 \%$ & $-3 \%$ \\
\hline \multirow{3}{*}{ Mult2 } & $1 \mathrm{M}$ & 0.92 & $+14 \%$ & $-5 \%$ & $-18 \%$ & $+33 \%$ & $-16 \%$ \\
\hline & $4 \mathrm{M}$ & 0.49 & $+84 \%$ & $+34 \%$ & $-64 \%$ & $+68 \%$ & $+2 \%$ \\
\hline & $16 \mathrm{M}$ & 0.22 & $+316 \%$ & $+202 \%$ & $-78 \%$ & $+170 \%$ & $-9 \%$ \\
\hline \multirow{3}{*}{ Mult2.2 } & IM & 0.96 & $+16 \%$ & $+10 \%$ & $-14 \%$ & $+38 \%$ & $-10 \%$ \\
\hline & $4 \mathrm{M}$ & 0.52 & $+84 \%$ & $+54 \%$ & $-52 \%$ & $+73 \%$ & $-1 \%$ \\
\hline & $16 \mathrm{M}$ & 0.25 & $+285 \%$ & $+221 \%$ & $+15 \%$ & $-161 \%$ & $-14 \%$ \\
\hline \multirow{3}{*}{ TV } & $1 \mathrm{M}$ & 2.14 & $+4 \%$ & $-2 \%$ & $-22 \%$ & $+32 \%$ & $-2 \%$ \\
\hline & $4 M$ & 1.53 & $+14 \%$ & $+6 \%$ & $+12 \%$ & $+39 \%$ & $-8 \%$ \\
\hline & $16 \mathrm{M}$ & 0.82 & $+99 \%$ & $+75 \%$ & $+195 \%$ & $+87 \%$ & $+32 \%$ \\
\hline \multirow{3}{*}{ Sor } & IM & 15.46 & $+0 \%$ & $-0 \%$ & $+0 \%$ & $-11 \%$ & $-0 \%$ \\
\hline & $4 \mathrm{M}$ & 8.57 & $+9 \%$ & $-1 \%$ & $-12 \%$ & $-8 \%$ & $-2 \%$ \\
\hline & $16 \mathrm{M}$ & 2.17 & $+158 \%$ & $+34 \%$ & $.81 \%$ & $-4 \%$ & $+60 \%$ \\
\hline \multirow{3}{*}{ Tree } & $\mathrm{IM}$ & 1.60 & $+11 \%$ & $-3 \%$ & $-9 \%$ & $+35 \%$ & $-6 \%$ \\
\hline & $4 \mathrm{M}$ & 0.41 & $+124 \%$ & $-5 \%$ & $-32 \%$ & $+70 \%$ & $+18 \%$ \\
\hline & $16 \mathrm{M}$ & 0.25 & $+263 \%$ & $+38 \%$ & $+83 \%$ & $+77 \%$ & $-17 \%$ \\
\hline \multirow{3}{*}{ Lin } & IM & 0.69 & $+26 \%$ & $+6 \%$ & $+9 \%$ & $+6 \%$ & $+21 \%$ \\
\hline & $4 \mathrm{M}$ & 0.02 & $+2763 \%$ & $+1322 \%$ & $+81 \%$ & $+778 \%$ & $+1797 \%$ \\
\hline & $16 \mathrm{M}$ & 0.01 & $+4648 \%$ & $+2248 \%$ & $--\%$ & $+873 \%$ & $+1037 \%$ \\
\hline
\end{tabular}

This table displays $B I+S_{S}$ for five cold-start techniques, eight traces, an interval length of 10 million instructions, and three four-way set-associative cache sizes $(1,4$, and 16 megabytes).

Since the sample mean for both random and systematic samples are unbiased estimates of the population mean, a sampling method yields a more accurate estimate of the population mean, if and only if the variance of its estimate is less than the variance of the alternative.

Thus, systematic samples obtained by the constant bits method yield more accurate estimates of $\mathrm{MPI}_{\text {true }}$ than random samples whenever (A1) divided by (A2) is greater than one. Empirical results displayed in Table III of Section III-A2 show that the ratio is usually greater than one, implying that constant bits samples are generally better than random samples.

We can get more intuition into why systematic samples might be better than random samples by examining the deriva- tion in [8, p. 208]. Using classical analysis of variance, he shows systematic sampling is more precise, if and only if:

$$
\begin{aligned}
\frac{1}{k(n-1)} & \sum_{j=1}^{k} \sum_{i=1}^{n}\left(\mathrm{mpi}_{j i}-\widehat{\operatorname{MPI}}_{S(j)}\right)^{2} \\
& >\frac{1}{s-1} \sum_{i=1}^{s}\left(\mathrm{mpi}_{i}-\text { MPI }_{\text {true }}\right)^{2} .
\end{aligned}
$$

where mpi ${ }_{j i}$ is the $i$ th member of the $j$ th systematic sample. In other words, systematic sampling more precisely estimates the mean of a population if the variance between observations within a systematic sample is greater than the population variance. Thus, we found that systematic samples obtained using constant bits were better than random samples, because systematically sampling sets captured more variation than was present in the population of all sets.

\section{APPENDIX B}

\section{COMPUTING CONFIDENCE INTERVALS}

In this appendix, we describe how we calculate the $90 \%$ confidence interval for a sample $\mathbf{S}$ containing $n$ MPI observations, $\mathrm{mpi}_{1}, \cdots, \mathrm{mpi}_{n}$. Since computing confidence intervals for systematic samples is complex [8, Section 8.11], we compute our confidence intervals by treating our systematic samples as random samples. Because our systematic samples estimate $\mathrm{MPI}_{\text {true }}$ with less variance than do random samples (Table III) the confidence intervals we calculate will tend be larger than necessary. Thus, if sample means are approximately normal, as they are for five of our eight traces (Section III-A2), $\mathrm{MPI}_{\text {true }}$ should lie within the $90 \%$ confidence intervals of more than $90 \%$ of the samples.

We first compute the MPI of sample $\mathbf{S}, \widehat{\mathrm{MPI}}_{S}$ with:

$$
\widehat{\mathrm{MPI}}_{S}=\frac{1}{n} \sum_{i=1}^{n} \mathrm{mpi}_{i},
$$

and estimate $\mathrm{MPI}_{S}$ 's standard deviation with:

$$
\begin{aligned}
\widehat{\mathrm{STD}}_{S}= & \sqrt{\frac{1}{n-1} \sum_{i=1}^{n}\left(\mathrm{mpi}_{i}-\widehat{\mathrm{MPI}}_{S}\right)^{2}} \\
& \times \frac{1}{\sqrt{n}} \times \sqrt{\frac{s-n}{s}} .
\end{aligned}
$$

$\widehat{\mathrm{STD}}_{S}$ is the product of three factors: 1) the sample standard deviation of the mpi ${ }_{i}$ 's, given that their true mean is unknown, 2) a $\frac{1}{\sqrt{n}}$ adjustment because $\widehat{\mathrm{MPI}}_{S}$ is the mean of the $n$ mpi $_{i}$ 's, and 3 ) a finite population correction factor $[8,(2.12)]$, which is important only when $n$, the sample size, is a substantial fraction of $s$, the population size. The $90 \%$ confidence interval for $\widehat{\mathrm{MPI}}_{S}$ is $\widehat{\mathrm{MPI}}_{S} \pm \widehat{\mathrm{STD}}_{S} \cdot t_{n-1}^{90 \%}$, where $t_{n-1}^{90 \%}$ is the value of the student-t statistic that has a tail of $5 \%$ (on each end) for $n-1$ degrees of freedom. We approximate the $t$-statistic with a normal for most our results, because $n$ is large $[8$, p. 27].

\section{APPENDIX C ADDITIONAL DATA}

In this appendix, we provide additional data to support the claims made in the body of the text. Figures 5(a) and (b) and 
Tables $\mathrm{X}$ and $\mathrm{XI}$ are more fully described in the body, where they are referenced.

\section{ACKNOWLEDGMENT}

We would like to thank The Western Research Laboratory of Digital Equipment Corporation, especially A. Borg and D. Wall, for the traces used in this study. J. Bartlett, R. De Leone, J. Dion, N. Jouppi, B. Mayo, and D. Stark all were a tremendous help in providing traceable applications. P. Vixie and C. Hawk helped to store the traces. P. Beebe and the Systems Lab were able to satisfy our enormous computing needs. M. Litzkow and M. Livny adapted Condor to the requirements of these simulations. $H$. Stone gave comments on an earlier version of this work, while S. Adve, V. Adve, G. Gibson and the anonymous referees scrutinized drafts of this paper.

\section{REFERENCES}

I1] A. Agarwal, J. Hennessy, and M. Horowitz, "Cache performance of operating system and multiprogramming workloads," ACM Trans. Computer Syst., vol. 6, no. 4, pp. 393-431, Nov. 1988

[2] A. Agarwal and M. Huffman, "Blocking: Exploiting spatial locality for trace compaction," Proc. Conf. Measurement and Modeling of Computer Systems 1990, pp. 48-57.

[3] A. Borg, R. E. Kessler, G. Lazana and D. W. Wall, "Long address traces from risc machines: Generation and analysis," Res. Rep. 89/14, Western Res. Lab., Digital Equipment Corp., Palo Alto, CA, Sept. 1989.

[4] A. Borg, R. E. Kessler. and D. W. Wall, "Generation and analysis of very long address traces," in Proc. 17th Annu. Int. Symp. Comput. Architecture, 1990, pp. 270-279.

[5] W. G. Cochran, Sampling Techniques, 3rd ed. New York: John Wiley, 1977.

[6] M. C. Easton and R. Fagin, "Cold-start vs. warm-start miss ratios," Commun. ACM, vol. 21, no. 10, pp. 866-872, Oct. 1978.

[7] P. Heidelberger and H. S. Stone, "Parallel trace-driven cache simulation by time partitioning," IBM Res. Rep. RC 15500 , no. 68960, Feb. 1990.

[8] M. D. Hill and A. J. Smith, "Evaluating associativity in CPU caches," IEEE Trans. Comput., vol. 38, no. 12, pp. 1612-1630, Dec. 1989

[9] N. P. Jouppi, "Improving direct-mapped cache performance by the addition of a small fully-associative cache and prefetch buffers," in Proc. 17th Annu. Int. Symp. Comput. Architecture, 1990, pp. 364-373.

[10] R. E. Kessler, "Analysis of multi-megabyte secondary CPU cache memories," Ph.D. thesis, Comput. Sci. Tech. Rep. no. 1032, Univ. of Wisconsin-Madison, WI, July 1991.

[11] D. Kroft, "Lockup-free instruction fetch/prefetch cache organization," in Proc. 8th Annu. Int. Symp. Comput. Architecture, 1981, pp. 81-87.

[12] S. Laha, J. H. Patel, and R. K. Iyer, "Accurate low-cost methods for performance evaluation of cache memory systems," IEEE Trans. Comput., vol. 37, no. 11, pp. 1325-1336, Nov. 1988.

[13] S. Laha, "Accurate low-cost methods for performance evaluation of cache memory systems," Ph.D. Thesis, Univ. of Illinois UrbanaChampaign, IL, 1988.

[14] I. Miller, J. E. Freund, and R. Johnson, Probability and Statistics for Engineers, fourth ed. Englewood Cliffs, NJ: Prentice Hall, 1990.

[15] M. J. K. Nielsen, "Titan system manual," Res. Rep. 86/1, Western Res. Lab., Digital Equipment Corp., Palo Alto, CA, Sept. 1986.

[16] S. A. Przybylski, "Performance-directed memory hierarchy design," Ph.D. thesis, Tech. Rep. CSL-TR-88-366, Stanford Univ., Stanford, CA, Sept. 1988

[17] S. Przybylski, M. Horowitz, and J. Hennessy, "Characteristics of performance-optimal multi-level cache hierarchies," in Proc. 16th Annu. Int. Symp. Comput. Architecture, 1989, pp. 114-121

[18] T. R. Puzak, "Analysis of cache replacement algorithms," Ph.D. thesis, Univ. of Massachusetts, Amherst, MA. Feb. 1985.

[19] A. D. Samples, "Mache: No-loss trace compaction," in Proc. Int. Conf. Measurement and Modeling of Comput. Sist., 1989, pp. 89-97.

[20] A. J. Smith, "Two methods for the efficient analysis of memory address trace data," IEEE Trans. Softuare Eng.. vol. SE-3, no. 1, pp. 94-101, Jan. 1977
[21] - "Cache memories," Computing Surveys, vol. 14, no. 3, pp 473-530, Sept. 1982

[22] H. S. Stone, High-Performance Computer Architecture, second ed. Reading, MA: Addison-Wesley, 1990

[23] W. Wang and J. Baer, "Efficient trace-driven simulation methods for cache performance analysis," in Proc. Conf. Measurement and Modeling of Comput. Sist., 1990, pp. 27-36.

[24] D. A. Wood, "The design and evaluation of in-cache address translation,” Ph.D. thesis, Comput. Sci. Division, Tech, Rep, UCB/CSD 90/565. Univ. of California, Berkeley, CA, Mar. 1990.

[25] D. A. Wood, M. D. Hill, and R. E. Kessler, "A model for estimating trace-sample miss ratios," in Proc. ACM SIGMETRICS Conf. Measurement and Modeling of Comput. Syst., 1991, pp. 79-89.

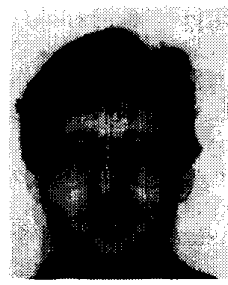

Richard E. Kessler (S'85-M'91) earned the B.S degree with high distinction in electrical and computer engineering from the University of Iowa, lowa City, in 1987, and the M.S. and Ph.D. degrees in Computer Science from the University of Wisconsin, Madison, in 1989 and 1991, respectively.

He is currently a Senior Architecture Engineer at Cray Research, Inc., Chippewa Falls, WI, specializing on the Massively-Parallel Processing (MPP) systems. His research interests include the architecture, operating systems, and performance analysis of computing systems. His recent research focuses largely on the architecture, analysis, and design of large-scale multiprocessing systems.

Dr. Kessler is a member of ACM

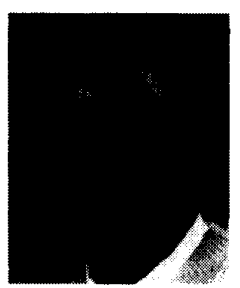

Mark D. Hill (S'81-M'87) earned the B.S.E. degree in computer engineering from the University of Michigan, Ann Arbor, in 1981, and the M.S. and Ph.D. degrees in computer science from the University of California, Berkeley, in 1983 and 1987 respectively.

$\mathrm{He}$ is currently an Assistant Professor in the Computer Sciences Department at the University of Wisconsin, Madison. He is interested in the design and evaluation of computer architectures. His recent work focuses on the memory systems of highperformance uniprocessors and shared-memory multiprocessors. J. R. Larus, D. A. Wood and he currently co-lead the ARPA-and NSF-sponsored Wisconsin Wind Tunnel Project that is exploring cost-effective and scalable support for shared memory in parallel supercomputers.

Dr. Hill is a 1989 recipient of the National Science Foundation's Presidential Young Investigator award and a member of ACM.



David A. Wood (S'81-M'90) received the B.S. degree in electrical engineering and computer science at the University of California, Berkeley, in 1981. After graduation, he worked on one of the earliest shared-memory multiprocessors at the Synapse Computer Corporation, where he helped develop a relational database system. He then returned to U.C. Berkeley, where he earned the Ph.D. degree in computer science in 1990 under Prof. R. Katz.

$\mathrm{He}$ is currently an Assistant Professor in the Computer Sciences and Electrical and Computer Engineering Departments at the University of Wisconsin, Madison. His interests range from VLSI design to operating systems, but focus on the design and evaluation of computer architectures, with an emphasis on memory systems for shared-memory multiprocessors. Together with M. D. Hill and J. R. Larus, he co-leads the NSF-sponsored Wisconsin Wind Tunnel Project that is exploring cost-effective and scalable support for shared memory in parallel supercomputers.

Dr. Wood is a 1991 recipient of the National Science Foundation's Presidential Young Investigator award and a member of the ACM. 